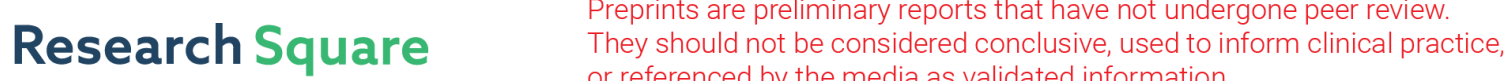 or referenced by the media as validated information. \\ Estimation of Model Accuracy By A Unique Set of Features And Tree-Based Regressor
}

\author{
Mor Bitton \\ Ben-Gurion University of the Negev \\ Chen Keasar ( $\nabla$ Keasar@bgu.ac.il ) \\ Ben-Gurion University of the Negev
}

\section{Research Article}

Keywords:

Posted Date: March 7th, 2022

DOI: https://doi.org/10.21203/rs.3.rs-1393722/v1

License: (c) (i) This work is licensed under a Creative Commons Attribution 4.0 International License. Read Full License 


\title{
Estimation of Model Accuracy by a unique set of features and tree-based regressor
}

\author{
Mor Bitton ${ }^{1}$ and Chen Keasar ${ }^{1, *}$ \\ ${ }^{1}$ Ben Gurion University, Department of Computer Science, Be'er Sheva, Israel \\ *Keasar@bgu.ac.il
}

\begin{abstract}
Computationally generated models of protein structures bridge the gap between the practically negligible price tag of sequencing and the high cost of experimental structure determination. By providing a low-cost (often free) partial alternative to experimentally determined structures, these models help biologists design and interpret their experiments. Obviously, the more accurate the models the more useful they are. However, methods for protein structure prediction generate many structural models (called decoys) of various qualities, necessitating means for the estimation of their accuracy.

Here we present MESHI_consensus, a new method for the estimation of model accuracy. The method uses a treebased regressor and a set of structural, target-based, and consensus-based features. The new method achieved high performance in the EMA (Estimation of Model Accuracy) track of the recent CASP14 community-wide experiment (https://predictioncenter.org/casp14/index.cgi). The tertiary structure prediction track of that experiment revealed an unprecedented leap in prediction performance by a single prediction group/method, namely AlphaFold2. This achievement would inevitably have a profound impact on the field of protein structure prediction, including the accuracy estimation sub-task. We conclude this manuscript with some speculations regarding the future role of accuracy estimation in a new era of accurate protein structure prediction.
\end{abstract}

\section{Introduction}

Protein structure prediction $(\boldsymbol{P S P})$ is a major challenge in computational biology. Given a target protein sequence (hereafter referred to as "target"), PSP methods aim to provide a three-dimensional model of the protein molecule. Such models help biologists build their theories and design their experiments. They provide a partial remedy to the high cost and much labor required for the experimental determination of structures. Typically, prediction methods generate many alternative structural models for each target. These models (hereafter referred to as "decoys") have diverse qualities even if generated by the same method, and often the best decoys of different targets are generated by different prediction methods. Unfortunately, large sets of alternative decoys do not provide much insight into biological problems, and the identification of the best decoys has been recognized early on ${ }^{1}$ as an essential PSP sub-task, known as Estimation of Model Accuracy $(\boldsymbol{E M A}$, aka $\boldsymbol{Q A})$.

This manuscript presents MESHI_consensus, a new EMA method, with state-of-the-art performance. Specifically, MESHI_consensus aims to predict the similarity of decoys to the corresponding native structures in terms of the zero to one Global Distance Test Total Score $\left(\boldsymbol{G D T} \boldsymbol{T}_{-} \boldsymbol{T S}\right)^{2}$, which assigns a score of one to decoys that are very similar to the native structure and lower scores to decoys that are less similar. A close to zero score indicates an irrelevant decoy.

For more than two decades already, the Critical Assessment of Protein Structure Prediction (CASP) biannual and communitywide, prediction experiments ${ }^{3-5}$ monitor the performance of prediction methods and accelerate their development. In each experiment, CASP organizers collect around a hundred targets at the final stages of their structural determination, and challenge researchers to submit blind predictions of the yet unknown structures. The assessment of these decoys, when the structures are finally determined, allows a reliable evaluation of prediction methods. The CASP experiments have several tracks for PSP sub-tasks, and since 2008, EMA is considered a CASP category ${ }^{6-9}$. CASP experiments play a two-fold role in the current study: our benchmark ${ }^{10}$ is based on decoys submitted to the $9^{\text {th }}$ to $13^{\text {th }}$ CASP rounds, and the $14^{\text {th }}$ experiment is used to evaluate the new EMA method.

EMA methods come in two flavors: local, assigning an accuracy measure to each residue of a model ${ }^{11-13}$, and global, assessing the qualities of complete models ${ }^{6}$. Often the former, in addition to its own merit, serves as a stepping stone to the latter ${ }^{14-16}$. Another major distinction between EMA methods is related to their input. For single-model methods ${ }^{17-19}$, the internal properties of a single decoy structure (e.g., estimated energy and compactness) are used to assess its quality. Multi-model (aka consensus) methods ${ }^{20,21}$ use multiple decoys of a target as input to estimate their accuracy. Structural similarity between independently generated decoys serves as an indication that they are likely to be all similar to the unknown native structure. MESHI_consensus is a global EMA method that combines single decoy properties and consensus information. 
Most recent EMA methods use machine learning $(\boldsymbol{M L})$ algorithms, including neural networks ${ }^{22-24}, \mathrm{SVM}^{25-27}$, and treebased models ${ }^{28}$, to create a statistical model that combines measurable features into a single number, which estimates decoy quality ${ }^{25,29}$. To this end, ML algorithms use datasets of annotated decoys and learn the intricate relations between the features and decoy quality. Specifically, the EMA methods use decoy structures to produce meaningful features, such as statistical pairwise potentials ${ }^{30,31}$ and consensus-derived terms ${ }^{32}$. These features constitute the input for regression models ${ }^{33,34}$ that integrate them into a single score. Our method, MESHI_consensus, uses a tree-based machine-learning algorithm to estimate decoy qualities from 982 features. An alternative ML approach was recently suggested by ${ }^{35}$ and ${ }^{13,36,37}$, who used deep convolutional $(\mathrm{CNN})$ and graph convolutional network $(\mathrm{GCN})$ respectively, to explicitly model the decoy structures and the favorable and adverse interactions that underlie the observed accuracy.

\section{Methods}

The following sections introduce the basic components of our method, dubbed MESHI_consensus. We first present our benchmark, a dataset of targets and decoys thereof from previous CASP experiments, and the features derived from them. Then we describe the performance measures that guided the development of the ML model, as well as the model design procedure, which includes regressor and hyper-parameters selection. Finally, we present a data filtering process that reduces training set noise.

\section{Decoys dataset}

We trained and evaluated our method using a dataset that consists of 73,053 single-chain decoys, which were generated as blind server predictions of 345 CASP9-CASP13 targets (2010-2018) (Table S1) an extension of the dataset used in ${ }^{10}$. These targets are a non-redundant subset of the $\approx 430$ targets of these CASP experiments. To this end, targets were considered redundant, and discarded, if a newer target was strictly similar by either sequence $\left(E-\right.$ value $\left.<10^{-3}\right)$ or structure (more than a half of the residues could be structurally aligned by the iterative magic fit method of Swiss-PDB-Viewer ${ }^{38,39}$ ). Duplicated decoys, having identical conformations as other decoys (typically from different servers of the same group), were identified and removed.

Many server-generated decoys include clashes (too short distances) between atoms and other structural distortions, such as deviations from correct bond lengths and angles. Thus, before feature extraction, each decoy is subjected to energy minimization using the MESHI molecular modeling package ${ }^{40}$. The energy function includes strong spatial constraints, and most distortions are removed with negligible structural changes.

The test set of this study includes 67 CASP14 targets (10,889 decoys), which were predicted by modeling servers during the CASP14 experiment (May-August, 2020). Both decoy generation and the estimation of their accuracy by MESHI_consensus web server were done in a blind fashion before their structures became available. After the decoys were downloaded from the CASP14 website they were energy minimized by MESHI package and their features were fed to the MESHI_consensus model.

\section{Features dataset}

In this study, each decoy is represented by a vector of 982 features. These features may be divided into two broad classes: decoy-features, and target-features that modulate the former.

Decoy-features: The following features are calculated using the MESHI package ${ }^{40}$.

- Basic features: 142 features derived solely from single decoy structures ${ }^{41}$. They include a mix of commonly used and novel knowledge-based energy terms (some of which unpublished yet). These terms include pair-wise atomic potentials $^{30,42,43}$, torsion angles ${ }^{44}$, hydrogen bonds, and hydrogen bond patterns ${ }^{45}$, solvation terms, "meta" energy terms that consider the distribution of other terms within the protein atoms, an extended radius of gyration that takes into account different classes of amino acids (polar vs. non-polar, secondary structure elements vs. coil region, etc.), and compatibility of the decoys with solvent exposure prediction ${ }^{46}$, and with 3-, 8- and 13-classes secondary structure predictions ${ }^{47-49}$.

- Consensus features: 7 features (equations 1-7) that represent the similarities between a decoy of a specific target and the other decoys of the same target. These terms are calculated as follows:

$\forall d \in T$, where $T$ is a decoy set of some target

$$
\begin{array}{r}
g d t_{i_{-}} \text {consensus }(d)=\frac{1}{|T|} \sum_{s \in T} g d t_{i}(d, s) \\
\text { where i } \in\{\mathbf{1}, \mathbf{2}, \mathbf{4}, \mathbf{8}\}
\end{array}
$$




$$
\begin{aligned}
& g d t_{-} t s_{-} \text {consensus }(d)=\frac{1}{|T|} \sum_{s \in T} g d t_{-} t s(d, s) \\
& g d t_{-} h a_{-} \text {consensus }(d)=\frac{1}{|T|} \sum_{s \in T} g d t_{-} h a(d, s) \\
& r m s_{-} \text {consensus }(d)=\frac{1}{|T|} \sum_{s \in T} R M S D(d, s)
\end{aligned}
$$

Where:

$$
\forall d, s \in T
$$

$$
\begin{aligned}
& g d t_{j \in\{0.5,1,2,4,8]}(d, s)=\text { The maximal fraction of } s \text { residues } \\
& \text { that are less than } j \AA \text { form the corresponding residues } \\
& \text { of } d \text { after superposition }{ }^{2} . \\
& g d t_{-} t s(d, s)=\frac{\sum_{i \in\{1,2,4,8\}} g d t_{i}(d, s)}{4} \\
& g d t_{-} h a(d, s)=\frac{\sum_{k \in\{0.5,1,2,4\}} g d t_{k}(d, s)}{4}
\end{aligned}
$$

Target-features: EMA datasets are organized in two levels; The objects that we study, and whose accuracies we predict are decoys. Yet each decoy belongs to a specific target (with no overlap between the targets). Each target is characterized by a unique sequence, which is shared by all its decoys, and a unique native structure. Thus, the mapping of features to decoy qualities may be biased by target characteristics such as length and chemical composition (amino acid sequence), which differ between targets but are typically identical in most decoys of a given target. Therefore, feature distributions and their relation to decoy quality differ between targets (Fig 1). Further, some training set targets may be less informative than others, with respect to certain features, due to specific characteristics such as ligand binding.

We use this domain knowledge to generate target-specific features that allow the learning process to modulate the outcome of the decoy features:

- One-hot encoding of target name: binary features (one per target). That is, for each target $T$ of the training set, there is a feature $O H_{T}$ such that $O H_{T}=1$ for all the decoys of $T$ and 0 otherwise. When positioned in a node of a decision tree, $O H_{T}$ splits the leaves of the sub-tree to $T$ and non $-T$, rendering the features in the nodes of the $T$ sub-tree practically meaningless. Interestingly the training process does make use of this ability to eliminate the effect of specific features in specific targets.

- Z-score, a normalized (zero mean and standard deviation of one) version of each basic feature, based on the target's mean and standard deviation.

- Amino acid composition:

- 20 features for the frequency of each amino acid in the sequence of the target.

- 6 features for the frequency of amino acids with certain properties in the sequence: Positive charged, negative charged, aromatic, polar, and non-polar.

Combining all the feature vectors of the decoys dataset creates a features dataset. 


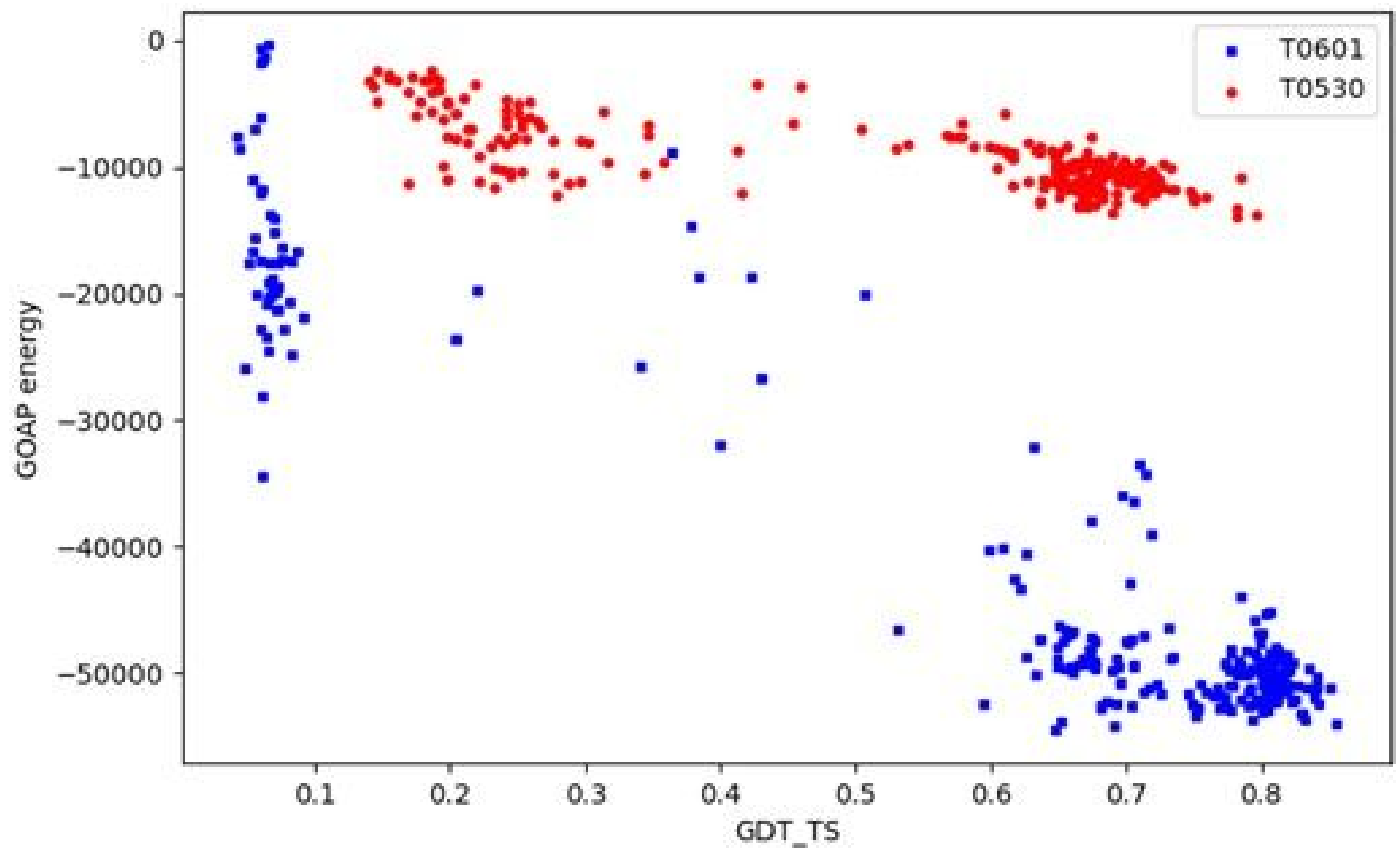

Figure 1. Target characteristics bias feature values. Each point in the figure represents the "GOAP" energy ${ }^{50}$ and accuracy (measured in GDT_TS between the decoys and the native structures, that is $g d t \_t s(d, n)$, Eq 6, where $n$ is the native structure of that target) of a single decoy. Decoys of the two CASP targets T0601 and T0530 are depicted by blue and red points respectively. "GOAP" energy term is a strong feature and it (anti) correlates well with the accuracies of both targets. Yet, given a feature value range (e.g., around -10000), the qualities of the two proteins are very different.

\section{Performance measures}

We aim at predicting the accuracy of decoys in terms of GDT_TS between the decoys and the native structures, that is $g d t_{-} t s(d, n)(\mathrm{Eq} 6)$ where $n$ is the native structure of that target. Specifically, we use three performance criteria:

1. Root mean square of prediction errors $(\boldsymbol{R M S E})$ - the per-target distance between the prediction values and the observed (true) values.

2. LOSS - for each target, the difference between the quality of the best decoy (highest observed GDT_TS) and the quality of the top-ranking decoy.

3. 5-LOSS - for each target, the minimum difference between the quality of the best decoy (highest observed GDT_TS) and the qualities of the five top-ranking decoys.

For dataset decoys, method performance is estimated by the median of 345 Leave-One-Target-Out cross-validation experiments (one per dataset target). In each experiment, the statistical model is trained using all the targets except one, which serves as the test set. This strategy is computationally expensive but reduces biases, and in a sense simulates the real-world scenario, where we learn from all the decoys of targets whose native structures are known and assess the quality of decoys of a yet structurally uncharacterized target.

\section{Model design}

The design of these EMA methods aimed to optimize two performance criteria: the median of the per-target RMSE and median LOSS. We used Leave-One-Target-Out cross-validation experiments to choose the regressor, its hyper-parameters, and the data filtering strategy. 


\section{Regressor}

In this work, we formulate EMA as a regression problem that maps measurable features of the decoys to a continuous quality score (GDT_TS) between the decoys and the native structures. To this end, we tested three regressors: linear regression, Light Gradient Boosted Machine (LightGBM) regressor ${ }^{51}$, and a fully connected neural network. The superior performance (Fig 2) of LightGBM motivated us to examine five other tree-based regressors: BaggingRegressor, GradientBoostingRegressor, RandomForestRegressor, and ExtraTreesRegressor from the scikit-learn library ${ }^{52}$, as well as Extreme Gradient Boosting (XGB) regressor $^{53}$. We remained with LightGBM, however, as it outperforms all five by a small margin and is faster to train.

(A)

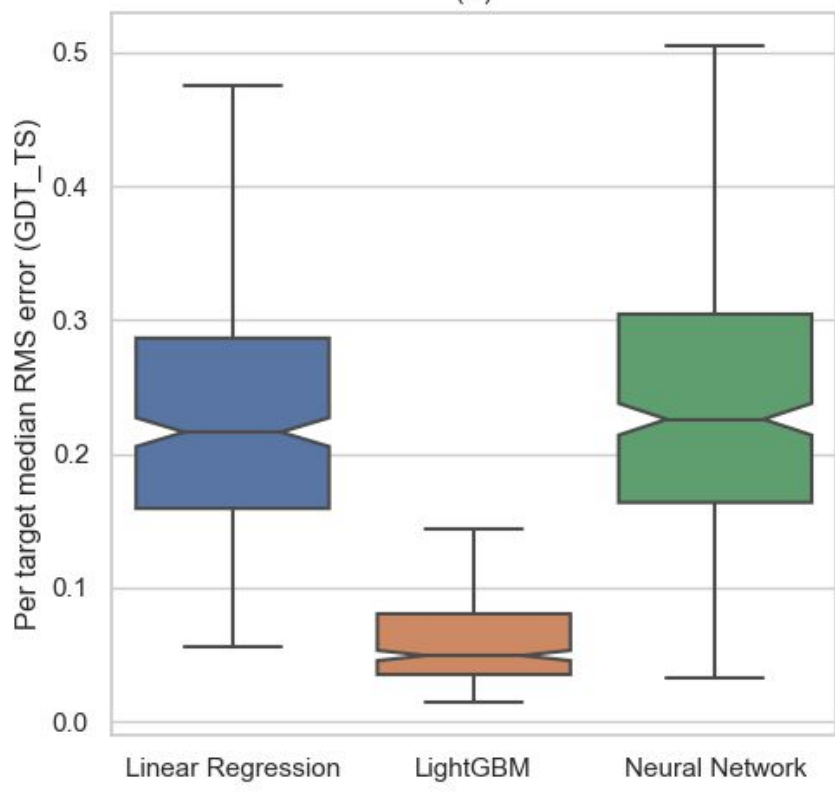

(B)

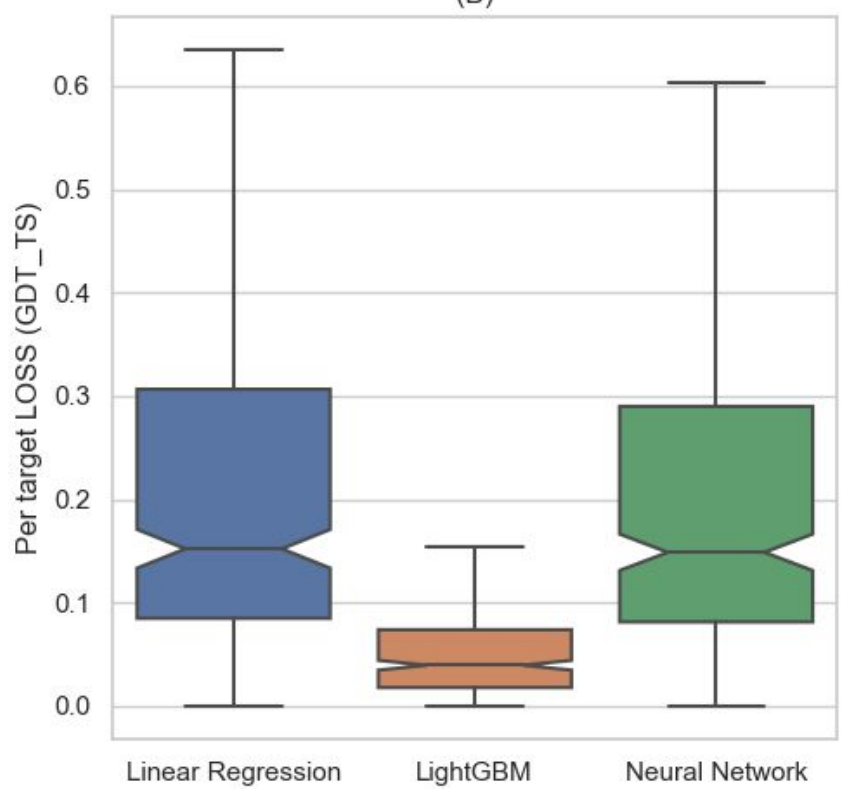

Figure 2. LightBGM ${ }^{51}$ outperforms linear regression and neural networks. The box plots depict the results of Leave-One-Target-Out experiments in terms of RMSE (A) and LOSS (B), using three different regressors. The difference in performance between LightBGM and the two other methods is statistically significant (Wilcoxon one-sided test with a p-value $<1.39 \mathrm{e}-51$ ). The performances of a few other tree-based methods were practically indistinguishable from LightBGM (data not shown) but computation time was much longer.

\section{Hyperparameters}

We used grid search to find the optimal values for two hyperparameters of LightGBM regressor: learning rate and the number of estimators. Learning rate 0.1 and 100 estimators achieved good results in a reasonable computation time. For all the other parameters, we used the default values as supplied by the LightGBM framework. Specifically, the loss function that the regressor training minimizes is the RMSE.

\section{Data filtering}

Some of the dataset targets are isolated chains of multi-subunit complexes (e.g., single helices of helix-bundles). Estimating their quality is a challenge due to hydrophobic interface residues that are superficially exposed when seen out of context. For such targets, MESHI may produce feature values that are inconsistent with the label (GDT_TS), increasing noise and impairing the learning process. The qualities of such targets are hard to estimate, even in an over-fitting scenario. The removal of 18 such targets (Table S1) from the training set significantly reduces the median error of quality estimates and does not affect the identification of the best decoys (Fig 3).

\section{Results}

MESHI_consensus was developed using a dataset of CASP server decoys that were generated as blind predictions in five consecutive CASP experiments (9-13). CASP14 decoys serve as the ultimate test set as their true qualities were unknown at the time of prediction. Here we present the method's performance in predicting the accuracies of dataset decoys, consider the contributions of different feature types, and conclude by presenting, and discussing CASP14 performance. 
(A)

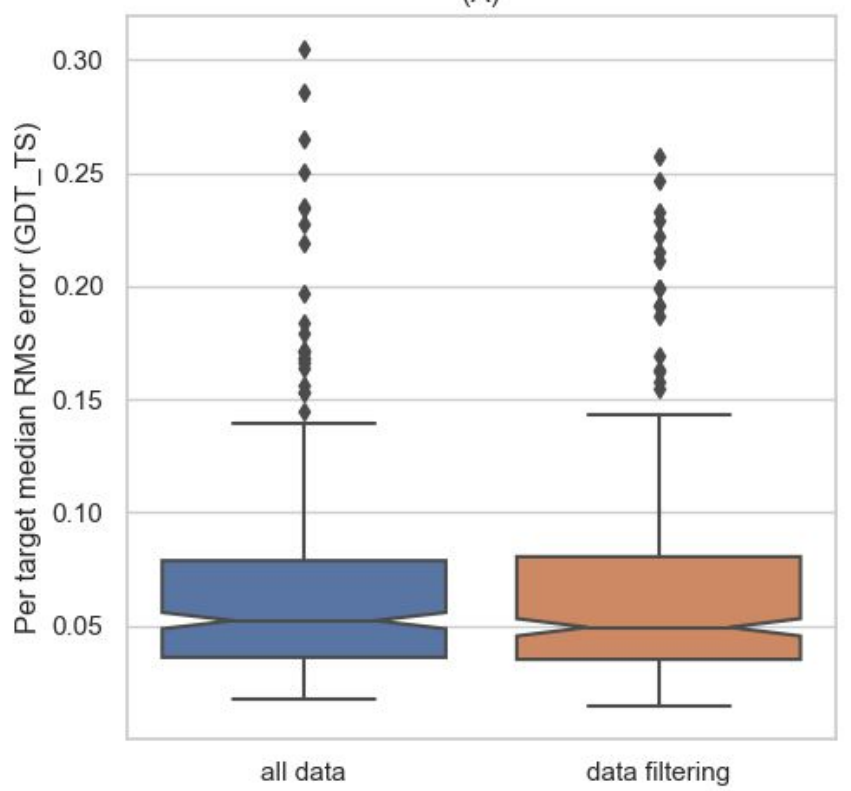

(B)

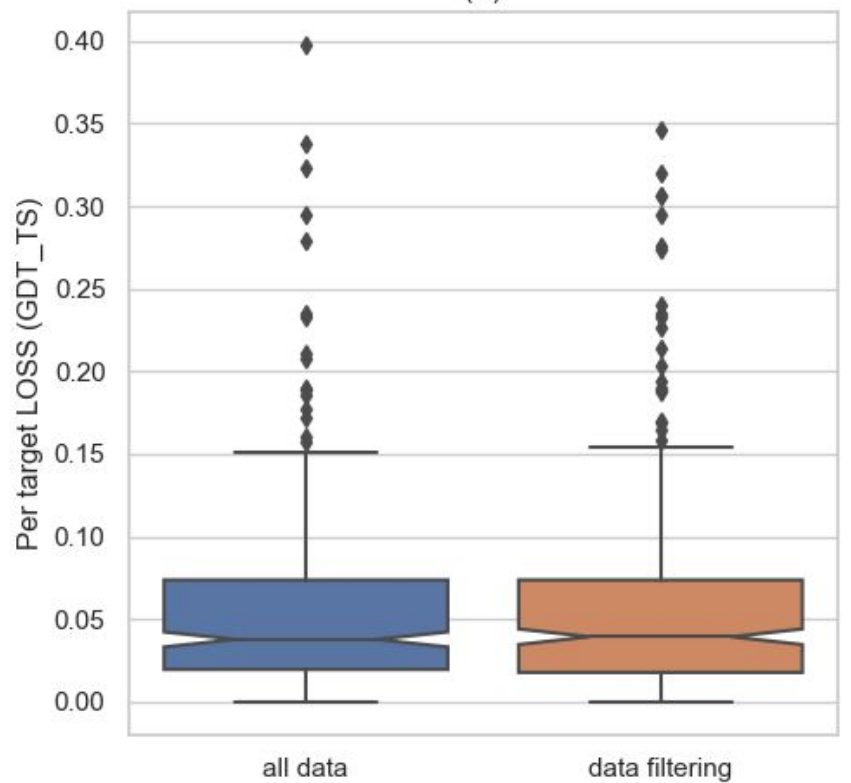

Figure 3. Filtering out outlier targets from the training set reduces the error in quality estimation (A), and does not affect the identification of the best decoys (B). The box plots depict the results of Leave-One-Target-Out experiments with and without data filtering in the training set. A - The median of the RMSE is significantly reduced by data filtering (Wilcoxon one-sided test, with a p-value of 0.005). B - data filtering does not affect the distribution of LOSS (the quality differences between the top-ranking decoy, and the best decoy in the set). Many of the worse performing outliers in the plots are proteins that were filtered out from the training set.

\section{Dataset Performance}

The performance of MESHI_consensus is estimated by Leave-One-Target-Out experiments on the dataset decoys. The qualities of about half of the targets are estimated well (Fig 4), with small $(<0.05)$ RMSE, and low $(<0.04)$ LOSS. A small fraction of the targets $(<6 \%)$ are practically missed, with RMSE or LOSS above 0.2 .

\section{Feature importance}

MESHI_consensus uses a large number of features for the estimation of decoy accuracies. To assess their contributions to the LBGM statistical model, we first checked the importance ranking of the basic features and all the features. Following the results (Fig 5), we also performed Leave-One-Target-Out experiments with only the basic features, only the consensus features, and with all the features (Fig 6). Additional subgroups of features were tested (data not shown), but the best result was obtained when we used the whole set of features. The measure we used for the importance of the features is "GAIN", which is the sum of the information gains of all splitting points that use that feature, where information gain is the Kullback-Leibler ${ }^{54}$ divergence of the data before and after the split. A higher value of this metric, when compared to another feature, implies it is more important for the predictive model.

The ten highest importance features, in LightGBM models that use either all the features or only the basic ones, are depicted in Fig 5. These models were trained on all the benchmark targets. Qualitatively similar estimates of feature GAINs, derived from benchmark subsets, are reported in Feature importance file (supplementary material). All but one of the LightGBM models make use of the possibility to distinguish between decoys from different targets. When target-specific features are available to the models, they choose from a wide variety, without a clear preference. The basic features were not intended to include such features explicitly, yet the models assign relatively high importance to the total number of side-chain atoms in the decoy. This feature seems almost arbitrary and was added to the basic features by mistake (being a component of other features). We speculate that the models consistently chose it as it allows target distinction. When all features are considered, consensus features are ranked highest by a large margin, apparently, because good (i.e, close to native) decoys are similar to one another (they are all similar to the native structure), while low-quality decoys can be very different from each other. This observation is consistent with the dominance of consensus-based methods in the EMA field. When only the basic features are considered, two classes of features become dominant. The first class (e.g., sasaCompatibility), quantifies the agreement 

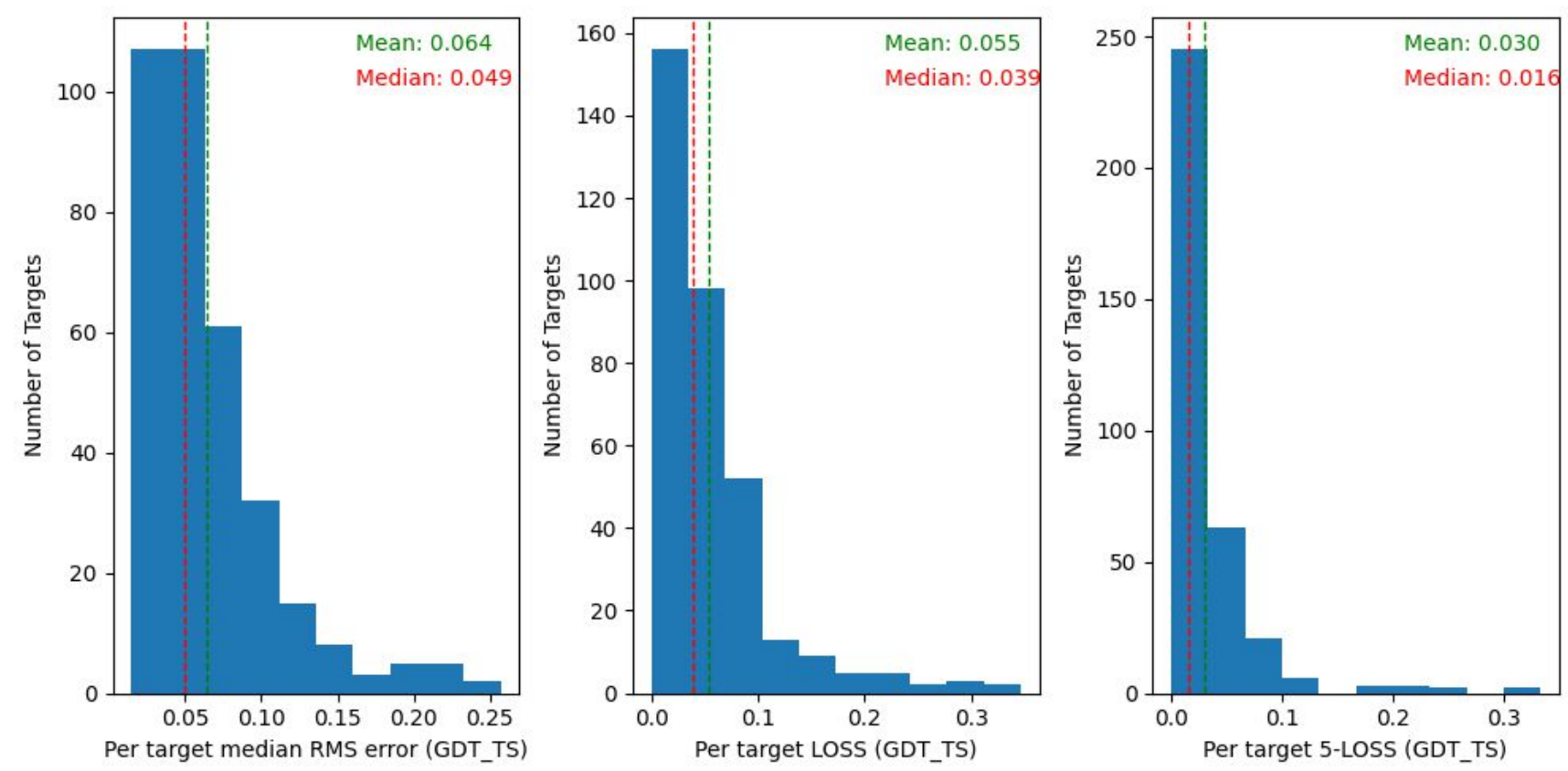

Figure 4. Benchmark performance of MESHI_consensus. The results of 345 Leave-one-target-out experiments are summarized in three histograms: (A) RMSE; (B) LOSS, the difference between the quality of the top-ranking decoy and the quality of the best one. (C) LOSS5, the minimal difference between the qualities of 5 top-ranking decoys and the quality of the best one. Median (red) and mean (green) values are indicated by vertical lines.

between the observed and predicted solvent exposed area and secondary structure of decoy residues. Apparently, an inability of a decoy to reproduce predicted solvent exposure and secondary structure is a strong indicator of low quality. This is consistent with the disruptive effect of out-of-context targets (e.g., isolated subunits) on learning. The second important class of basic features (e.g., goap_ag) rewards structural traits that are common in native structures. One common trait is compactness which manifests itself by a large number of atom contacts. The other common trait is the abundance of specific atomic interactions (e.g., contacting side-chain atoms of hydrophobic residues). Having many favorable interactions, and a compact structure are strong indicators of high decoy quality. Finally, as our quality measure, GDT_TS between the decoys and the native structures represents the fraction of accurately modeled residues, it is bound by the fraction of the target sequence which is actually modeled. Coverage features depict this fraction and are consistently ranked among the ten most important.

Considering the dominance of consensus features, we tested whether the other features are needed at all. To this end, we performed a Leave-One-Target-Out experiment with three different sets of features (Fig 6). The first experiment served as a baseline and included only the basic features. The second experiment used only the consensus features and the third used all the features. In both, the RMSE and LOSS, the best results are obtained by using the entire set of features. For RMSE (A), which is the loss function of the regressor, most of the improvement is due to the consensus features, consistent with the "GAIN" results (Fig 5). Yet by using all the features we obtain statistically better performance. For LOSS (B), the basic features outperform the consensus ones, reflecting the difficulty of consensus features to identify exceptionally good decoys. The best decoy of target T0581 for example (BAKER-ROSETTASERVER_TS4, 0.64 GDT_TS) was picked by the model trained with the basic features only. The model that was trained with all the features picked the second-best decoy (GDT_TS 0.33), which is similar to some other inaccurate decoys. Notably, however, adding consensus features to the basic ones reduces the number of outliers and the magnitude of their deviation from the median performance. This is probably because consensus features are indifferent to complex subunits and membrane proteins that distort many basic features.

\section{CASP14: MESHI_consensus}

The last round of CASP experiments, CASP14 (May-August, 2020) serves as the independent test set of this study. MESHI_consensus took part in that experiment as an EMA server and submitted 11,135 global quality predictions of server decoys. One target, T1093, was missed due to technical failure. Figure 7 depicts the best (left) and worst (right) results with targets T1046s2 and T1031 respectively. The overall performance on this test set (Fig 8) is comparable to that of the benchmark (Fig 4). The slight performance reduction is discussed below. Comparison with the other 71 research groups that competed in the EMA category 
reveals a state-of-the-art performance, with more than $65 \%$ of the predictions ranked within the top 10 in either LOSS or RMSE. Specifically, MESHI_consensus reached:

- Top GDT_TS MCC(50) score (Table 1)

(https://predictioncenter.org/casp14/qa_aucmcc.cgi)

- Third lowest average prediction error (Table 2)

(https://predictioncenter.org/casp14/qa_diff_mqas.cgi.

- Sixths lowest average LOSS (Table 3)

(https://predictioncenter.org/casp14/qa_diff2best.cgi).

Notably, among the other top-performing methods, one (MESHI) is a MESHI_consensus variant, which is discussed below.

\begin{tabular}{l|l} 
Group name & MCC(50) \\
\hline MESHI_consensus & 0.746 \\
MESHI & 0.742 \\
DAVIS-EMAconsensus & 0.728 \\
ModFOLDclust2 & 0.724 \\
MUfoldQA_G & 0.723 \\
EMAP_CHAE & 0.707 \\
UOSHAN & 0.696 \\
Yang_TBM & 0.692
\end{tabular}

Table 1.

\begin{tabular}{l|l} 
Group name & RMSE \\
\hline DAVIS-EMAconsensus & 0.0673 \\
MUfoldQA_G & 0.0723 \\
MESHI_consensus & 0.0724 \\
MESHI & 0.0725 \\
ModFOLDclust2 & 0.0735 \\
EMAP_CHAE & 0.0739 \\
Yang_TBM & 0.0804 \\
UOSHAN & 0.0836
\end{tabular}

Table 2.

\begin{tabular}{l|l} 
Group name & LOSS \\
\hline MULTICOM-CONSTRUCT & 0.0735 \\
MULTICOM-AI & 0.0792 \\
MESHI & 0.0793 \\
MULTICOM-CLUSTER & 0.0802 \\
MUfoldQA_G & 0.082 \\
MESHI_consensus & 0.084 \\
BAKER-ROSETTASERVER & 0.084 \\
BAKER-experimental & 0.0845
\end{tabular}

Table 3.

CASP14 performances. The tables present the top-scoring groups ${ }^{58-63}$ by three measures: MCC(50) (Table 1), RMSE (Table 2), and LOSS (Table 3). Results are reproduced from the CASP website at (https://predictioncenter.org/casp14). Note that in the CASP site RMSE and LOSS are referred to as "differences (predicted vs observed)" and "difference from the best", respectively, and their performances are depicted as percentages. The servers "Seder2020" and "Seder2020hard" that submitted an EMA prediction for a single target were omitted. 


\section{Discussion}

This manuscript introduces MESHI_consensus, a new method for quality assessment of protein decoys. MESHI_consensus uses a large and diverse set of features, representing both physical concepts (e.g., energy terms) and domain knowledge (target-specific and consensus-based features). The features are integrated to a single score by a powerful and computationally efficient, tree-based LightGBM regressor ${ }^{51}$. One type of state-of-the-art features, which the method misses, is compatibility with predicted distances derived from multiple sequence alignments $(\boldsymbol{M S A})^{15,64}$. MSA-driven distances are the keystone of the current breakthrough in PSP, and compatibility with them seems to be a powerful feature ${ }^{9}$.

The development of MESHI_consensus was guided by Leave-One-Target-Out experiments using a non-redundant dataset of decoys from previous CASP experiments. The recent CASP14 provided an objective performance test, and MESHI_consensus scores among the top methods (see Results). Yet, notwithstanding these achievements, the overall performance (Fig 8), is worse than in the dataset's Leave-One-Target-Out experiments (Fig 4). Specifically, for six targets MESHI_consensus has a LOSS value above 0.2, the LOSS value of one of them, T1031, reached as high (and bad) as 0.4 (as shown in Fig 7, right). This target, as well as four others: T1035, T1039, T1040, T1043 are domains of a large protein, Phage DNA-dependent RNA polymerase (PDB code: 6vr4). The structure of this protein was solved intact and these domains have numerous inter-domain interactions that are missing in the decoys, exposing large hydrophobic patches (Fig 9).

Such patches are typically indicators of low accuracy. A similar phenomenon is also observed in the dataset (see the Data filtering section). Unfortunately, we cannot comment at this stage on the sixth unsuccessful target, T1093, as its structure has not been released yet. Another, more speculative explanation for the lower performance is the methodological turning point of CASP14 (discussed below). It raises a major challenge to MESHI_consensus, as well as to any supervised learning method that uses CASP decoy sets for training. The test (CASP14 decoys) and training sets were not sampled from the same distribution, as the decoys of CASP14 are on average more accurate than those of previous CASP experiments. For the sake of completeness we should also mention the EMA predictions of MESHI group in CASP14, which was a curiosity-driven variant of MESHI_consensus, and ranked a bit higher. That variant simply adds server names as a feature, and will not be discussed further.

During the study, we invested much effort in analyzing failures, that is targets for which we considerably missed the actual decoy qualities and/or their rankings. Many of these failures could be rationalized in retrospect as related to the inability of our features to consider stabilizing inter-molecular interactions. We tried to implement insights from this analysis through data filtering with limited success. One may hope though that a more systematic approach to this problem may lead to better performance in future studies.

A profound limitation of MESHI_consensus is the reliance on a single native structure as the gold standard for the labeling of the data. This is in line with the common practice in the field, which is applied in CASP and in all the studies that we are aware of. Yet, this practice ignores the structural flexibility of proteins as manifested by diverse structures of the same protein in different PDB entries ${ }^{65}$, and in the results of NMR studies. This structural multiplicity can be introduced into the training phase of EMA methods if decoys are evaluated by their similarity to the closest of the known structures, rather than to a single arbitrary one.

Finally, CASP14 has witnessed a remarkable breakthrough in PSP, with many models of hard targets reaching experimental quality. This achievement had a limited effect on the EMA section of CASP14 as the cutting-edge method, AlphaFold ${ }^{66}$, did not provide a server. Yet, it is evident that a new standard of model qualities is set ${ }^{67}$. Will EMA be needed at all when the models are "almost perfect"? An obvious answer is that we are in the middle of the event and its consequences cannot be predicted. More fundamentally, the new achievements seem to open new horizons for PSP, considerably improving our ability to cope with essential problems like structures of molecular complexes and protein dynamics. These challenges require their own EMA tools. We believe that the insights, as well as specific features and machine learning techniques, gained in EMA studies of single-chain targets, will be the basis for EMA methods that cope with the new challenges.

\section{References}

1. Ginalski, K., Elofsson, A., Fischer, D. \& Rychlewski, L. 3D-Jury: a simple approach to improve protein structure predictions. Bioinformatics 19, 1015-1018, DOI: 10.1093/bioinformatics/btg124 (2003). https://academic.oup.com/ bioinformatics/article-pdf/19/8/1015/642841/btg124.pdf.

2. Zemla, A. LGA: a method for finding 3D similarities in protein structures. Nucleic Acids Res. 31, 3370-3374, DOI: 10.1093/nar/gkg571 (2003). Number: 13.

3. Moult, J. A decade of CASP: progress, bottlenecks and prognosis in protein structure prediction. Curr. Opin. Struct. Biol. 15, 285-289, DOI: 10.1016/j.sbi.2005.05.011 (2005).

4. Moult, J., Fidelis, K., Kryshtafovych, A., Rost, B. \& Tramontano, A. Critical assessment of methods of protein structure prediction—Round VIII. Proteins: Struct. Funct. Bioinforma. 77, 1-4, DOI: 10.1002/prot.22589 (2009). Number: S9. 
5. Moult, J., Fidelis, K., Kryshtafovych, A., Schwede, T. \& Tramontano, A. Critical assessment of methods of protein structure prediction (CASP) — round x. Proteins: Struct. Funct. Bioinforma. 82, 1-6, DOI: 10.1002/prot.24452 (2014). _eprint: https://onlinelibrary.wiley.com/doi/pdf/10.1002/prot.24452.

6. Wallner, B. \& Elofsson, A. Prediction of global and local model quality in CASP7 using Pcons and ProQ. Proteins: Struct. Funct. Bioinforma. 69, 184-193, DOI: 10.1002/prot.21774 (2007). Number: S8.

7. Kryshtafovych, A. et al. Assessment of the assessment: Evaluation of the model quality estimates in CASP10. Proteins: Struct. Funct. Bioinforma. 82, 112-126, DOI: 10.1002/prot.24347 (2014). _eprint: https://onlinelibrary.wiley.com/doi/pdf/10.1002/prot.24347.

8. Kryshtafovych, A., Monastyrskyy, B., Fidelis, K., Schwede, T. \& Tramontano, A. Assessment of model accuracy estimations in CASP12. Proteins: Struct. Funct. Bioinforma. 86, 345-360, DOI: 10.1002/prot.25371 (2018). Number: S1.

9. Cheng, J. et al. Estimation of model accuracy in casp13. Proteins: Struct. Funct. Bioinforma. 87, 1361-1377 (2019).

10. Sidi, T. \& Keasar, C. Loss-functions matter, on optimizing score functions for the estimation of protein models accuracy. bioRxiv 651349 (2019).

11. Wallner, B. \& Elofsson, A. Identification of correct regions in protein models using structural, alignment, and consensus information. Protein Sci. 15, 900-913 (2006).

12. Studer, G., Biasini, M. \& Schwede, T. Assessing the local structural quality of transmembrane protein models using statistical potentials (qmeanbrane). Bioinformatics 30, i505-i511 (2014).

13. Takei, Y. \& Ishida, T. P3cmqa: Single-model quality assessment using $3 \mathrm{~d} c n n$ with profile-based features. Bioengineering 8, 40 (2021).

14. Uziela, K. \& Wallner, B. Proq2: estimation of model accuracy implemented in rosetta. Bioinformatics 32, 1411-1413 (2016).

15. Maghrabi, A. H. \& McGuffin, L. J. Modfold6: an accurate web server for the global and local quality estimation of $3 \mathrm{~d}$ protein models. Nucleic acids research 45, W416-W421 (2017).

16. Olechnovic, K. \& Venclovas, C. Voromqa: Assessment of protein structure quality using interatomic contact areas. Proteins: Struct. Funct. Bioinforma. 85, 1131-1145, DOI: https://doi.org/10.1002/prot.25278 (2017). https://onlinelibrary. wiley.com/doi/pdf/10.1002/prot.25278.

17. Wallner, B. \& Elofsson, A. Can correct protein models be identified? Protein science 12, 1073-1086 (2003).

18. Olechnovič, K. \& Venclovas, Č. Voromqa: Assessment of protein structure quality using interatomic contact areas. Proteins: Struct. Funct. Bioinforma. 85, 1131-1145 (2017).

19. Ray, A., Lindahl, E. \& Wallner, B. Improved model quality assessment using proq2. BMC bioinformatics 13, 1-12 (2012).

20. Lundström, J., Rychlewski, L., Bujnicki, J. \& Elofsson, A. Pcons: A neural-network-based consensus predictor that improves fold recognition. Protein Sci. 10, 2354-2362 (2001).

21. Ginalski, K., Elofsson, A., Fischer, D. \& Rychlewski, L. 3d-jury: a simple approach to improve protein structure predictions. Bioinformatics 19, 1015-1018 (2003).

22. Faraggi, E. \& Kloczkowski, A. A global machine learning based scoring function for protein structure prediction. Proteins: Struct. Funct. Bioinforma. 82, 752-759, DOI: 10.1002/prot.24454 (2014). _eprint: https://onlinelibrary.wiley.com/doi/pdf/10.1002/prot.24454.

23. Zhang, J. \& Xu, D. Fast algorithm for population-based protein structural model analysis. PROTEOMICS 13, 221-229, DOI: 10.1002/pmic.201200334 (2013). Number: 2.

24. Terashi, G., Nakamura, Y., Shimoyama, H. \& Takeda-Shitaka, M. Quality assessment methods for 3d protein structure models based on a residue-residue distance matrix prediction. Chem. Pharm. Bull. 62, 744-753 (2014).

25. Qiu, J., Sheffler, W., Baker, D. \& Noble, W. S. Ranking predicted protein structures with support vector regression. Proteins: Struct. Funct. Bioinforma. 71, 1175-1182, DOI: 10.1002/prot.21809 (2008). Number: 3.

26. Manavalan, B. \& Lee, J. SVMQA: support-vector-machine-based protein single-model quality assessment. Bioinformatics 33, 2496-2503, DOI: 10.1093/bioinformatics/btx222 (2017). Number: 16.

27. Hippe, K., Lilley, C., Berkenpas, W., Kishaba, K. \& Cao, R. Zoomqa: Residue-level single-model qa support vector machine utilizing sequential and $3 \mathrm{~d}$ structural features. bioRxiv (2021). 
28. Manavalan, B., Lee, J. \& Lee, J. Random forest-based protein model quality assessment (rfmqa) using structural features and potential energy terms. PloS one 9, e106542 (2014).

29. Wang, Z., Tegge, A. N. \& Cheng, J. Evaluating the absolute quality of a single protein model using structural features and support vector machines. Proteins: Struct. Funct. Bioinforma. 75, 638-647, DOI: 10.1002/prot.22275 (2009). Number: 3.

30. Zhou, H. \& Skolnick, J. GOAP: a generalized orientation-dependent, all-atom statistical potential for protein structure prediction. Biophys. J. 101, 2043 - 2052 (2011). Number: 8.

31. Zhang, J. \& Zhang, Y. A novel side-chain orientation dependent potential derived from random-walk reference state for protein fold selection and structure prediction. PloS one 5, e15386 (2010).

32. Lundström, J., Rychlewski, L., Bujnicki, J. \& Elofsson, A. Pcons: A neural-network-based consensus predictor that improves fold recognition. Protein Sci. 10, 2354-2362, DOI: 10.1110/ps.08501 (2001). Number: 11.

33. Mirzaei, S., Sidi, T., Keasar, C. \& Crivelli, S. Purely structural protein scoring functions using support vector machine and ensemble learning. IEEE/ACM Transactions on Comput. Biol. Bioinforma. 16, 1515-1523, DOI: 10.1109/TCBB.2016. 2602269 (2019).

34. Korovnik, M. et al. Synthqa-hierarchical machine learning-based protein quality assessment. bioRxiv (2021).

35. Derevyanko, G., Grudinin, S., Bengio, Y. \& Lamoureux, G. Deep convolutional networks for quality assessment of protein folds. Bioinformatics 34, 4046-4053, DOI: 10.1093/bioinformatics/bty494 (2018). https://academic.oup.com/ bioinformatics/article-pdf/34/23/4046/26676600/bty494.pdf.

36. Sanyal, S., Anishchenko, I., Dagar, A., Baker, D. \& Talukdar, P. Proteingcn: Protein model quality assessment using graph convolutional networks. bioRxiv DOI: 10.1101/2020.04.06.028266 (2020). https://www.biorxiv.org/content/early/2020/04/ 07/2020.04.06.028266.full.pdf.

37. Shuvo, M. H., Bhattacharya, S. \& Bhattacharya, D. Qdeep: distance-based protein model quality estimation by residue-level ensemble error classifications using stacked deep residual neural networks. Bioinformatics 36, i285-i291 (2020).

38. Kaplan, W. \& Littlejohn, T. G. Swiss-pdb viewer (deep view). Briefings bioinformatics 2, 195-197 (2001).

39. Guex, N. \& Peitsch, M. C. Swiss-model and the swiss-pdb viewer: an environment for comparative protein modeling. electrophoresis 18, 2714-2723 (1997).

40. Kalisman, N. et al. MESHI: a new library of Java classes for molecular modeling. Bioinformatics 21, 3931-3932, DOI: 10.1093/bioinformatics/bti630 (2005). Number: 20.

41. Elofsson, A. et al. Methods for estimation of model accuracy in CASP12. Proteins: Struct. Funct. Bioinforma. 86, 361-373, DOI: 10.1002/prot.25395 (2018). Number: S1.

42. Samudrala, R. \& Moult, J. An all-atom distance-dependent conditional probability discriminatory function for protein structure prediction. J. Mol. Biol. 275, 895-916, DOI: 10.1006/jmbi.1997.1479 (1998). Number: 5.

43. Summa, C. M. \& Levitt, M. Near-native structure refinement using in vacuo energy minimization. Proc. Natl. Acad. Sci. 104, 3177-3182, DOI: 10.1073/pnas.0611593104 (2007). Number: 9.

44. Amir, E.-A. D., Kalisman, N. \& Keasar, C. Differentiable, multi-dimensional, knowledge-based energy terms for torsion angle probabilities and propensities. Proteins: Struct. Funct. Bioinforma. 72, 62-73, DOI: 10.1002/prot.21896 (2008). Number: 1.

45. Levy-Moonshine, A., Amir, E.-a. D. \& Keasar, C. Enhancement of beta-sheet assembly by cooperative hydrogen bonds potential. Bioinformatics 25, 2639-2645, DOI: 10.1093/bioinformatics/btp449 (2009). Number: 20.

46. Cheng, J., Randall, A. Z., Sweredoski, M. J. \& Baldi, P. SCRATCH: a protein structure and structural feature prediction server. Nucleic Acids Res. 33, W72-W76, DOI: 10.1093/nar/gki396 (2005).

47. Wang, S., Li, W., Liu, S. \& Xu, J. RaptorX-Property: a web server for protein structure property prediction. Nucleic Acids Res. 44, W430-W435, DOI: 10.1093/nar/gkw306 (2016). Number: W1.

48. McGuffin, L. J., Bryson, K. \& Jones, D. T. The PSIPRED protein structure prediction server. Bioinformatics 16, 404-405, DOI: 10.1093/bioinformatics/16.4.404 (2000). Number: 4.

49. Sidi, T. \& Keasar, C. Redundancy-weighting the pdb for detailed secondary structure prediction using deep-learning models. Bioinformatics (2020).

50. Zhou, H. \& Skolnick, J. GOAP: A Generalized Orientation-Dependent, All-Atom Statistical Potential for Protein Structure Prediction. Biophys. J. 101, 2043-2052, DOI: 10.1016/j.bpj.2011.09.012 (2011). Number: 8. 
51. Ke, G. et al. LightGBM: A Highly Efficient Gradient Boosting Decision Tree. In Guyon, I. et al. (eds.) Advances in Neural Information Processing Systems 30, 3146-3154 (Curran Associates, Inc., 2017).

52. Pedregosa, F. et al. Scikit-learn: Machine learning in python. J. machine Learn. research 12, 2825-2830 (2011).

53. Chen, T., He, T., Benesty, M., Khotilovich, V. \& Tang, Y. Xgboost: extreme gradient boosting. R package version 0.4-2 $1-4$ (2015).

54. Kullback, S. Information theory and statistics (Courier Corporation, 1997).

55. Kabsch, W. \& Sander, C. Dictionary of protein secondary structure: Pattern recognition of hydrogen-bonded and geometrical features. Biopolymers 22, 2577-2637, DOI: 10.1002/bip.360221211 (1983). Number: 12.

56. Wang, S., Weng, S., Ma, J. \& Tang, Q. DeepCNF-D: Predicting Protein Order/Disorder Regions by Weighted Deep Convolutional Neural Fields. Int. J. Mol. Sci. 16, 17315-17330, DOI: 10.3390/ijms160817315 (2015). Number: 8.

57. Levy-Moonshine, A., Amir, E.-a. D. \& Keasar, C. Enhancement of beta-sheet assembly by cooperative hydrogen bonds potential. Bioinformatics 25, 2639-2645 (2009).

58. Chen, X. et al. Protein model accuracy estimation empowered by deep learning and inter-residue distance prediction in casp14. Sci. Reports 11, 1-12 (2021).

59. Kryshtafovych, A. et al. Assessment of the assessment: evaluation of the model quality estimates in casp10. Proteins: Struct. Funct. Bioinforma. 82, 112-126 (2014).

60. Wang, W., Wang, J., Li, Z., Xu, D. \& Shang, Y. Mufoldqa_g: High-accuracy protein model qa via retraining and transformation. Comput. Struct. Biotechnol. J. 19, 6282-6290 (2021).

61. McGuffin, L. J. \& Roche, D. B. Rapid model quality assessment for protein structure predictions using the comparison of multiple models without structural alignments. Bioinformatics 26, 182-188 (2010).

62. Hiranuma, N. et al. Improved protein structure refinement guided by deep learning based accuracy estimation. Nat. communications 12, 1-11 (2021).

63. Ye, L. et al. Improved estimation of model quality using predicted inter-residue distance. Bioinformatics 37, 3752-3759 (2021).

64. Hou, J., Wu, T., Cao, R. \& Cheng, J. Protein tertiary structure modeling driven by deep learning and contact distance prediction in CASP13. Proteins: Struct. Funct. Bioinforma. 87, 1165-1178, DOI: https://doi.org/10.1002/prot.25697 (2019). _eprint: https://onlinelibrary.wiley.com/doi/pdf/10.1002/prot.25697.

65. Kosloff, M. \& Kolodny, R. Sequence-similar, structure-dissimilar protein pairs in the pdb. Proteins: Struct. Funct. Bioinforma. 71, 891-902, DOI: https://doi.org/10.1002/prot.21770 (2008). https://onlinelibrary.wiley.com/doi/pdf/10.1002/ prot.21770.

66. Jumper, J. et al. Highly accurate protein structure prediction with AlphaFold. Nature 1-11, DOI: 10.1038/ s41586-021-03819-2 (2021). Bandiera_abtest: a Cg_type: Nature Research Journals Primary_atype: Research Publisher: Nature Publishing Group Subject_term: Computational biophysics;Machine learning;Protein structure predictions;Structural biology Subject_term_id: computational-biophysics;machine-learning;protein-structurepredictions;structural-biology.

67. Baek, M. et al. Accurate prediction of protein structures and interactions using a three-track neural network. Science DOI: 10.1126/science.abj8754 (2021). Publisher: American Association for the Advancement of Science Section: Research Article.

\section{Acknowledgements}

The authors are grateful to Tomer Sidi for creating the benchmark used in this study, to Ilya Kaufman for fruitful discussions, and to Ofir Ezrielev for the careful reviewing.

\section{Author contributions statement}

C.K. conceived this study, supervised it, and curated the training data. M.B designed the experiments, performed them, and collected and analyzed the results. The manuscript was jointly written by both authors. 


\section{Additional information}

Table S1 Targets list. List of targets and corresponding CASP experiments. Targets marked in red are excluded from the training set.

Feature importance file Qualitatively estimates of feature GAINS, derived from benchmark subsets.

\section{Data availability}

The data sets generated and analysed during the current study are available at http://meshi1.cs.bgu.ac.il/BittonAndKeasar2021/ 


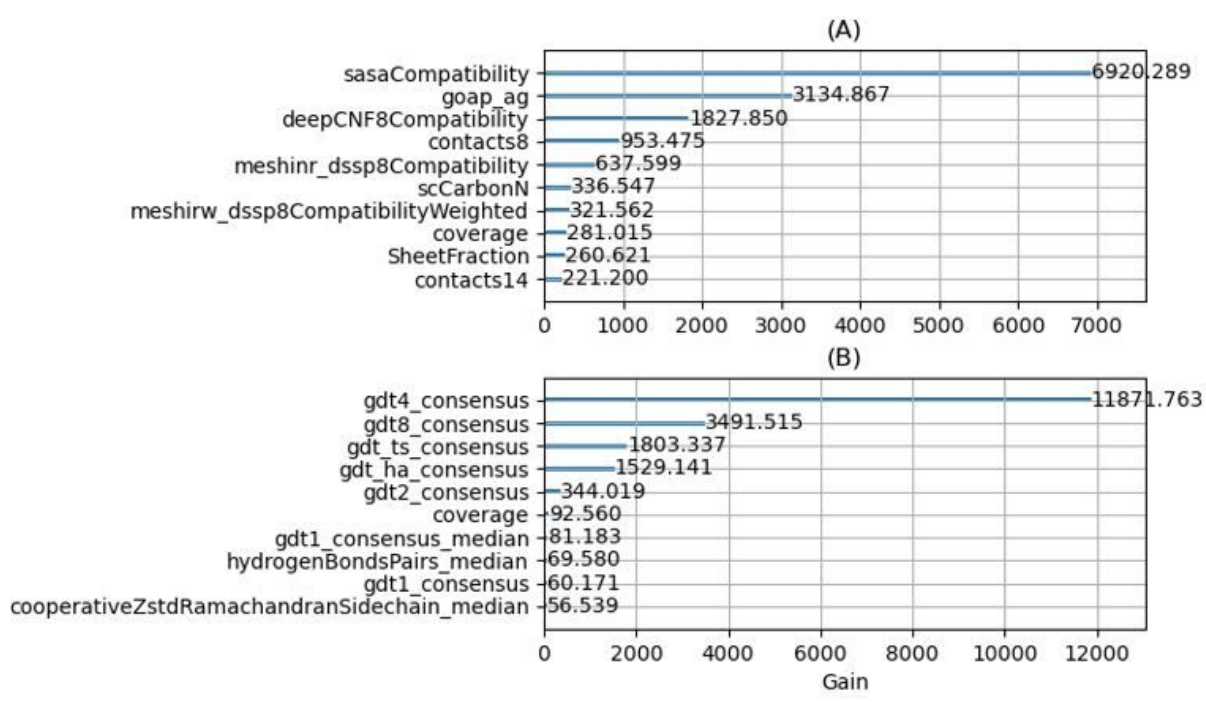

Figure 5. The ten most important (highest "GAIN") features considering only the basic features (A) and all features (B). The features from top to bottom:

- sasaCompatibility - a measure of the agreement between the solvent accessible surface area of the decoy's residues (as measured by $\operatorname{DSSP}^{55}$ ) and their predicted accessibility ${ }^{46}$.

- goap_ag - a pairwise orientation-dependent knowledge-based potential ${ }^{30}$.

- deepCNF8Compatibility - a measure of the agreement between the secondary structure ( 8 states) of the decoy's residues (as measured by DSSP ${ }^{55}$ ) and their predicted secondary structure ${ }^{56}$.

- contacts8 and contacts14 - the average numbers of contacts with thresholds of $8 \AA$ and $14 \AA$, respectively, between carbon atoms.

- meshinr_dssp8Compatibility and meshirw_dssp8Compatibility_Weighted - Two slightly different measures of the agreement between the secondary structure ( 8 states) of the decoy's residues (as measured by DSSP ${ }^{55}$ ) and their predicted secondary structure ${ }^{49}$.

- scCarbonN - the number of carbon atoms in the decoy's side-chains.

- coverage - The fraction of the target sequence, which the decoy models.

- SheetFraction - the fraction of beta-sheet resides within the residues with any secondary structure.

- consensus features - see Eq 1-4.

- gdt1_consensus_median - the median value of gdt1_consensus, among all the decoys of a specific target.

- hydrogenBondsPairs_median - the median value of a cooperative hydrogen bonds energy term ${ }^{57}$ among all the decoys of a specific target.

- cooperativeZstdRamachandranSidechain_median - the median value of a cooperative torsion angle energy term, among all the decoys of a specific target. 
(A)

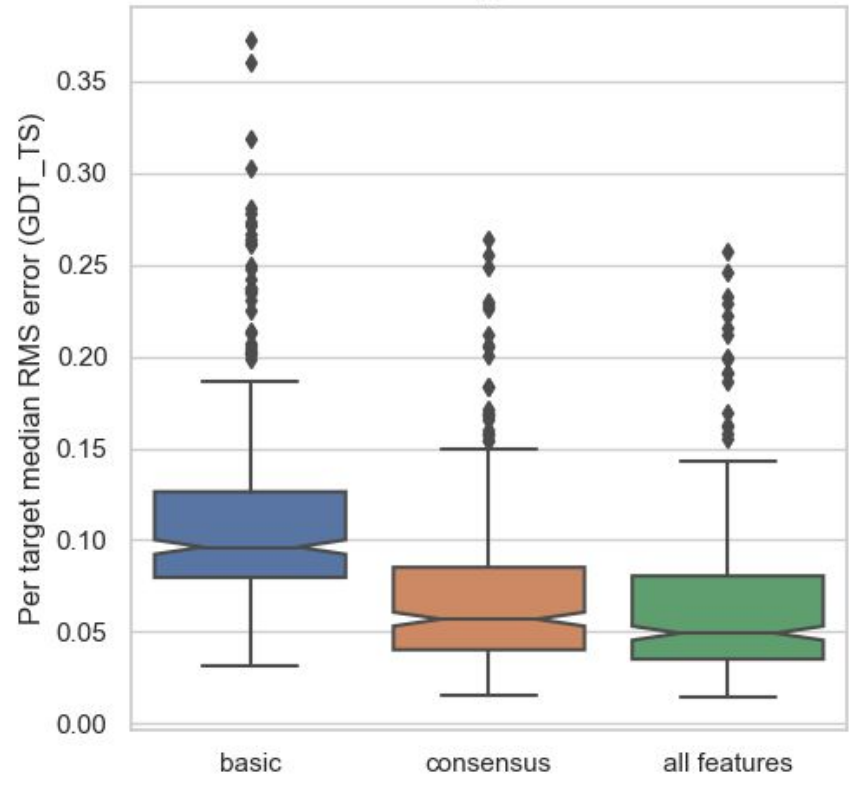

(B)

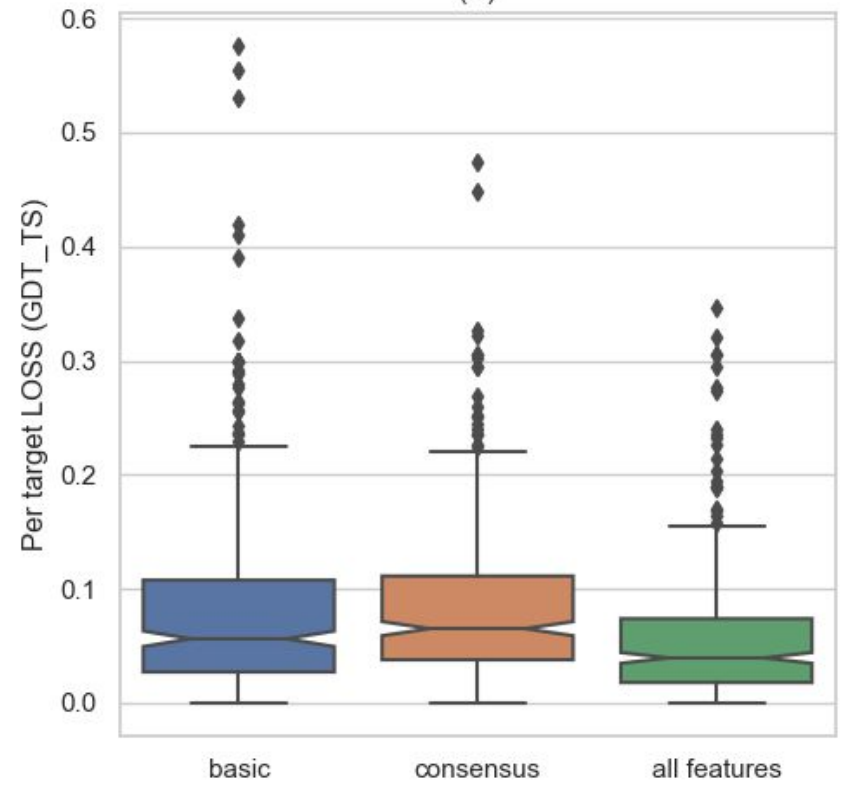

Figure 6. Per-target performance with different feature sets. The box plots show the per-target performance in terms of RMSE (A) and LOSS (B), of Leave-One-Target-Out experiments with the basic features (blue), consensus features (orange), and all features (green). The differences between the median performances of all the features and the median performances of the feature subsets are statistically significant (Wilcoxon one-sided test with a p-value $<10 \mathrm{e}-4$ ).
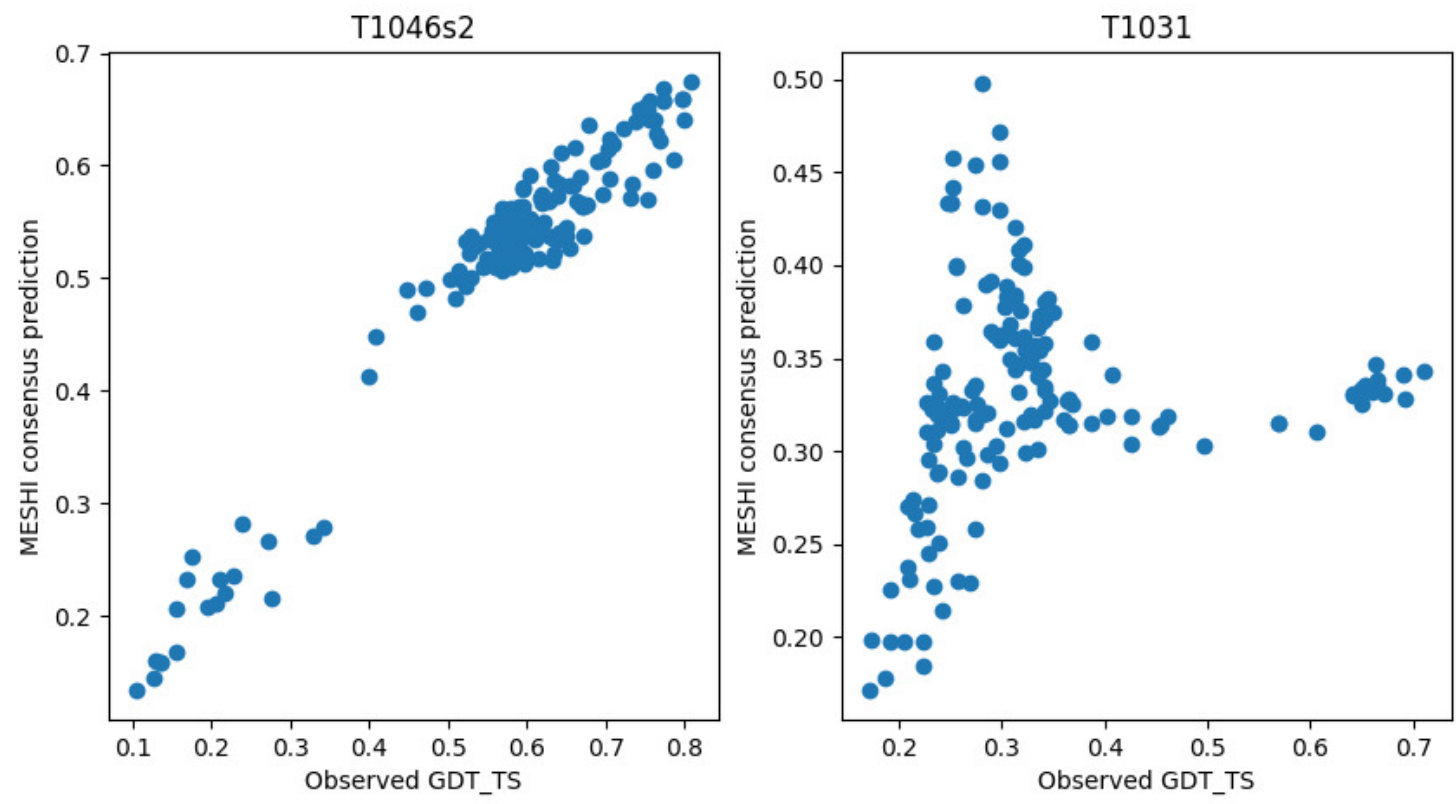

Figure 7. Examples of MESHI_consensus success and failure in CASP14. Predicted vs. observed qualities of decoys from two targets:

Left: For target T1046s2 (PDB code: 6px4), MESHI_consensus reached a low RMSE (0.074) and the top-scoring decoy is indeed the best one (zero LOSS).

Right: For target T1031 (PDB code: 6vr4) the RMSE is 0.128 and the best decoy ranked very low (LOSS is 0.428 ) 

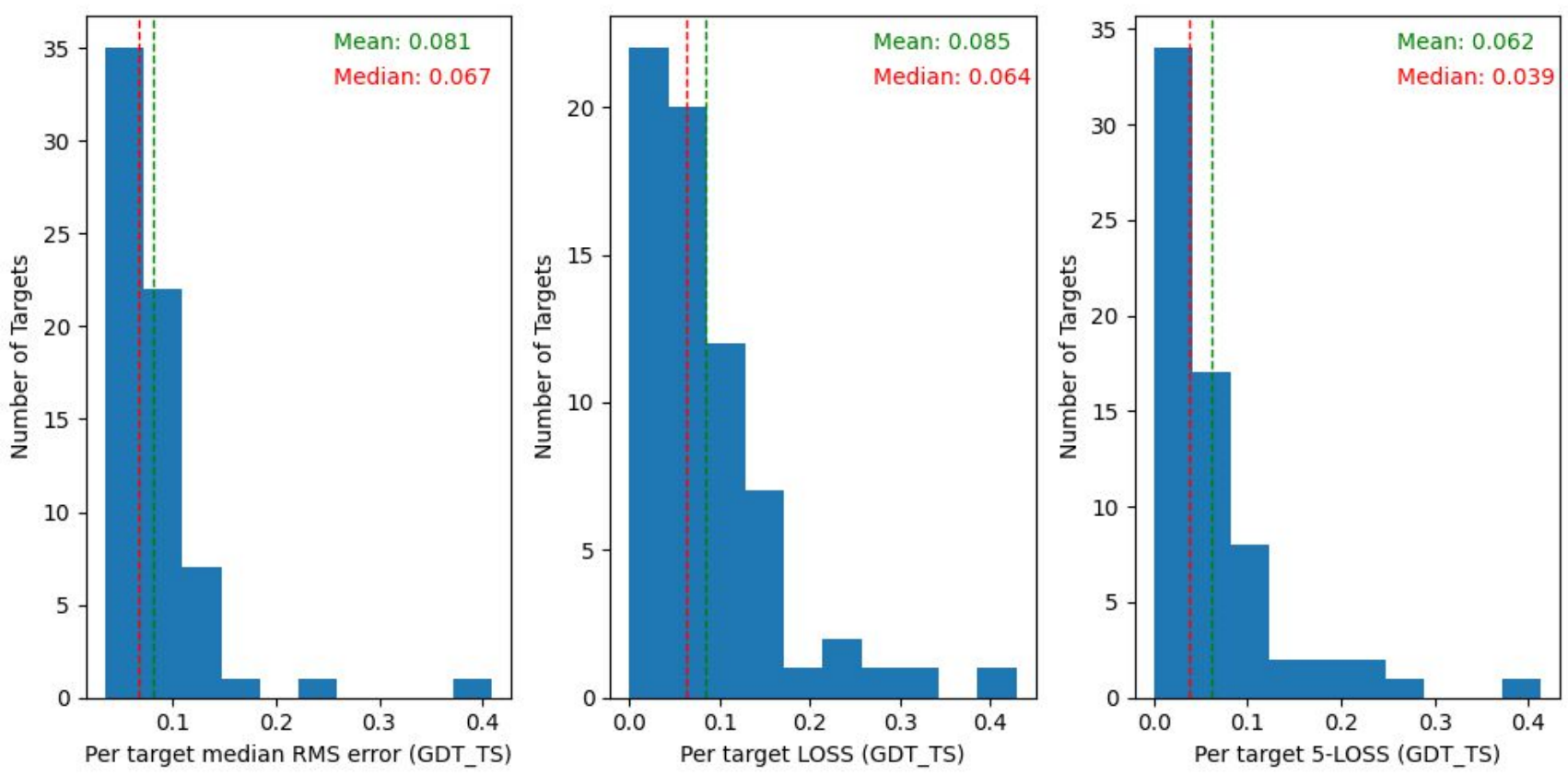

Figure 8. The decoy accuracies of most $(>\mathbf{5 0 \%})$ of the CASP14 targets can be predicted within an error of 0.07 GDT_TS units. The plots depict the results of MESHI_consensus method in CASP14. Median (red) and mean (green) values are indicated by vertical lines. 


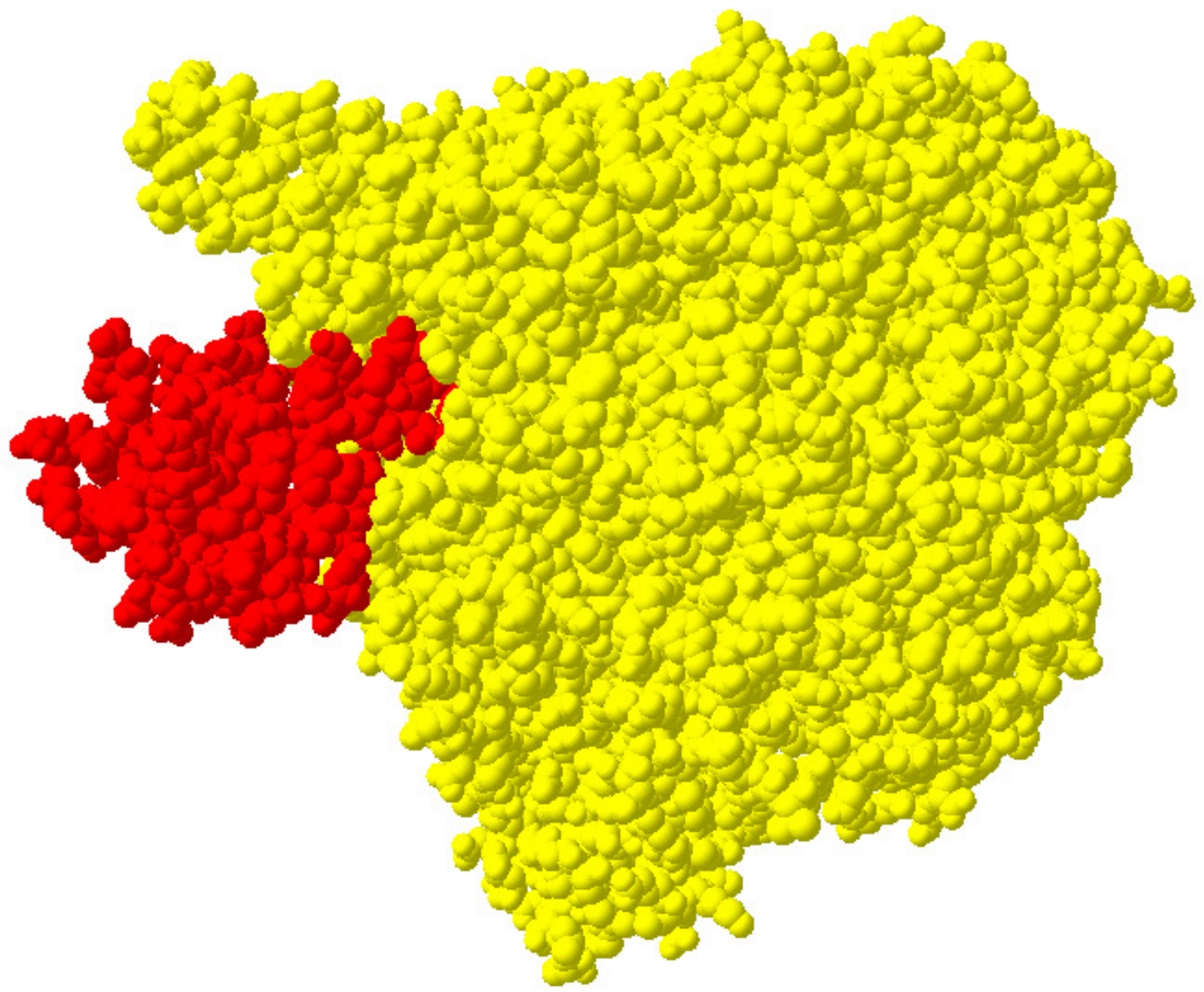

Figure 9. space-filling depiction of target T1031 in context. Target T1031 (red) consists of residues 1-95 of phi14:2 RNA polymerase (PDB code $6 \mathrm{vr} 4$, yellow). The domain has many contacts with the rest of the protein, and we speculate that the poor performance of MESHI_consensus with this target is due to the inter-domain interfaces, which are superficially exposed in the decoys. 


\section{Supplementary Files}

This is a list of supplementary files associated with this preprint. Click to download.

- Featureimportancefile.pdf

- Targetslist.pdf 